\title{
The Invar tensor package: Differential invariants of Riemann
}

\author{
J.M. Martín-García ${ }^{a}$, D. Yllanes ${ }^{\text {a }}$ \\ ${ }^{a}$ Instituto de Estructura de la Materia, CSIC, \\ C/ Serrano 123, Madrid 28006, Spain \\ R. Portugal ${ }^{b, c}$ \\ ${ }^{\mathrm{b}}$ Department of Applied Mathematics, University of Waterloo, \\ Waterloo, Ontario, Canada \\ ${ }^{\mathrm{c}}$ Laboratório Nacional de Computação Científica (LNCC), \\ Av. Getúlio Vargas 333, Petrópolis, RJ, CEP 25651-075, Brazil
}

\begin{abstract}
The long standing problem of the relations among the scalar invariants of the Riemann tensor is computationally solved for all $6 \cdot 10^{23}$ objects with up to 12 derivatives of the metric. This covers cases ranging from products of up to 6 undifferentiated Riemann tensors to cases with up to 10 covariant derivatives of a single Riemann. We extend our computer algebra system Invar to produce within seconds a canonical form for any of those objects in terms of a basis. The process is as follows: (1) an invariant is converted in real time into a canonical form with respect to the permutation symmetries of the Riemann tensor; (2) Invar reads a database of more than $6 \cdot 10^{5}$ relations and applies those coming from the cyclic symmetry of the Riemann tensor; (3) then applies the relations coming from the Bianchi identity, (4) the relations coming from commutations of covariant derivatives, (5) the dimensionallydependent identities for dimension 4, and finally (6) simplifies invariants that can be expressed as product of dual invariants. Invar runs on top of the tensor computer algebra systems xTensor (for Mathematica) and Canon (for Maple).
\end{abstract}

Key words: Riemann tensor, tensor calculus, Mathematica, Maple, computer algebra

PACS: 02.70.Wz, 04.20.-q, 02.40.Ky 


\section{Program summary}

Title of program: Invar Tensor Package v. 2.0

Catalogue identifier:

Program obtainable from: (submitted to Computer Physics Communications)

http://www. Incc.br/ portugal/Invar.html (Maple version) and

http://metric.iem.csic.es/Martin-Garcia/xAct/Invar/ (Mathematica)

Reference in $C P C$ to previous version: Computer Physics Communications 177 (2007) 640-648

Catalogue identifier of previous version: ADSP

Does the new version supersede the original program?: Yes. The previous version (1.0) only handled algebraic invariants. The current version (2.0) has been extended to cover differential invariants as well.

Computers: Any computer running Mathematica versions 5.0 to 6.0 or Maple versions 9 to 11

Operating systems under which the new version has been tested: Linux, Unix, Windows XP, MacOS

Programming language: Mathematica and Maple

Memory required to execute with typical data: $100 \mathrm{Mb}$

No. of bits in a word: 64 or 32

No. of processors used: 1

No. of bytes in distributed program, including test data, etc.: Code $<1 \mathrm{Mb}$; database: $40 \mathrm{Mb} ; 13$ expanded cases order 12 at commutation step: $250 \mathrm{Mb}$.

Distribution format: Unencoded compressed tar file

Nature of physical problem: Manipulation and simplification of scalar polynomial expressions formed from the Riemann tensor and its covariant derivatives.

Method of solution: Algorithms of computational group theory to simplify expressions with tensors that obey permutation symmetries. Tables of syzygies of the scalar invariants of the Riemann tensor.

Restrictions on the complexity of the problem: The present version only handles scalars, but not expressions with free indices.

Typical running time: One second to fully reduce any monomial of the Riemann tensor up to degree 7 or order 10 in terms of independent invariants.

\section{Introduction}

Extracting information from the Riemann curvature tensor of a metric field on a manifold is not an easy task due to its complicated algebraic structure. The problem can be handled when restricting to the algebraic invariants of the Riemann tensor, for which the problem of giving all of them in terms of a basis has been recently solved, after decades of continued work $[1,2,3]$. However, this is frequently not enough and the differential invariants of Rie- 
mann are then required. For example, Bonnor [4] has shown that the acceleration in the 'photon rocket' metric does not affect the algebraic Riemann invariants, but changes the differential invariants. In this way it is possible to introduce singularities in the curvature which are unnoticed by the algebraic curvature invariants [5]. Differential invariants of Riemann are also required to compute the different loop-orders of renormalization of the Einstein-Hilbert Lagrangian [6], or in dealing with its generalizations, like the Lagrangian $L\left(R, \nabla^{2} R, \ldots \nabla^{2 n} R\right)$ proposed in [7], or the general diffeomorphism invariant Lagrangian $L\left(g_{a b}, R_{b c d e}, \nabla_{a_{1}} R_{b c d e}, \ldots, \nabla_{\left(a_{1}\right.} \ldots \nabla_{\left.a_{m}\right)} R_{b c d e}, \ldots\right)$ analyzed in [8].

In spite of the large amount of effort dedicated to the algebraic invariants, very little has been said about how to manipulate large families of differential invariants, except for the important work by Fulling et al. [9], because the problem is much more complicated. Here we shall apply and generalize the techniques we proposed in [1] to cover this case, extending our tensor computer algebra system Invar to handle both the algebraic and the differential invariants of the Riemann tensor.

\section{The problem}

As in [1] we shall work on a manifold of dimension $d$ with a metric field $g_{a b}$ and its associated structures: the (torsionless) Levi-Civita connection $\nabla_{a}$, its Riemann tensor $R_{a b c d}$ and (when restricting to $d=4$ ) the totally antisymmetric tensor $\epsilon_{a b c d}$, such that $\nabla_{e} g_{a b}=0$ and $\nabla_{e} \epsilon_{a b c d}=0$.

Our main objective is constructing a basis of independent invariants (that is, scalars formed from contraction of several Riemann tensors and their $\nabla$ derivatives) and dual invariants (those also having an $\epsilon_{a b c d}$ tensor), along with all polynomial relations giving any other dependent invariant in terms of those in the basis. The restricted algebraic case without derivatives of Riemann has been essentially solved, both from the computational point of view [1] and, after a long series of contributions, from the theoretical point of view $[2,3]$. The general case is much more complicated and remains, however, nearly unexplored. The only article known to us in this direction is the work by Fulling et al. [9] in which, using Young tableaux techniques, they were able to compute numbers of independent nondual invariants in the basis for a variety of cases, in different dimensions. They also gave the members of the basis for the simpler of those cases, but no expansions of the dependent invariants were provided, rendering the result unpractical. This article fills in that important gap by recomputing the basis of invariants (hence confirming for the first time the results in [9]) together with a database of expressions of any other independent invariant in terms of the basis. 


\section{Notations}

Following Fulling et al. [9] we separate the set of all monomial invariants of degree $n$ (the number of Riemann tensors) in subsets $\mathcal{R}_{\left\{\lambda_{1}, \ldots, \lambda_{n}\right\}}$, where $\lambda_{i}$ is the differentiation order of the $i$-th Riemann tensor, assuming the tensors have been sorted such that $\lambda_{i} \leq \lambda_{i+1}$. An $n$-tuple $\left\{\lambda_{1}, \ldots, \lambda_{n}\right\}$ will be referred to as a case, with the corresponding invariants having $N=4 n+\sum_{i=1}^{n} \lambda_{i}$ indices and order $\Lambda=2 n+\sum_{i=1}^{n} \lambda_{i}$, the number of derivatives of the metric, not to be confused with the total number of derivatives of the Riemann tensors, which is $\sum_{i=1}^{n} \lambda_{i}$. Both $N$ and $\Lambda$ are always even numbers because we only consider scalar expressions, with all indices paired among them. For example an invariant of the case $\{0,1,3\}$, hence with $N=16$ indices and order $\Lambda=10$, is

$$
R_{a b c d} \nabla_{e} R^{e c f g} \nabla^{a} \nabla_{f} \nabla_{h} R_{g}^{b d h}
$$

Sets of dual invariants and dual cases will be denoted with an asterisk, as in

$\mathcal{R}_{\left\{\lambda_{1}, \ldots, \lambda_{n}\right\}}^{*}$ or $\left\{\lambda_{1}, \ldots, \lambda_{n}\right\}^{*}$. The algebraic cases considered in [1] are denoted here as $\{0, . \stackrel{n}{.}, 0\}$ for degree $n$.

We shall see that commutation of derivatives produces relations among invariants of different cases, because it converts second derivatives into additional Riemann tensors. This operation changes both the degree $n$ and the differentiation orders $\lambda_{i}$ of the Riemann tensors, but not the total order $\Lambda$ of metric derivatives. This allows a simple classification of the relations, which are all homogeneous in $\Lambda$. Following again ref. [9] we give results up to $\Lambda=12$, which consistently fills the gap among the algebraic cases of degrees 1 to 7 (that is $\Lambda=2$ to $\Lambda=14$ ) in [1]. Concerning duals, we give results up to $\Lambda=8$, also consistent with the algebraic degrees 1 to 5 of [1]. See tables 1, 2, 3 for the actual list of cases considered in this investigation, already sorted by their corresponding values of $\Lambda$ : there are 48 nondual cases and 15 dual cases to treat. In the following, when talking about total numbers of invariants and relations, we always include the 7 nondual and 5 dual algebraic cases already considered in [1].

\section{Algorithms}

Relations among Riemann invariants are a consequence of the symmetries obeyed by the Riemann tensor and its $\nabla$-derivatives. There are six different symmetries we can exploit and so we proceed in six respective consecutive steps, each producing new relations and therefore decreasing the number of 
independent invariants, as shown in tables 1, 2 and 3 (note that steps 3 and 4 are new with respect to [1]):

(1) Permutation symmetries. The Riemann tensor obeys the following symmetries under permutations of indices:

$$
R_{b a c d}=-R_{a b c d}, \quad R_{c d a b}=R_{a b c d}
$$

As explained in [1] this type of tensor symmetry, and the induced symmetries in tensor products, can be efficiently handled using fast algorithms for manipulation of permutation groups [10]. In our system, each monomial is converted in real time to its canonical form. For example, the canonical form of invariant (1) is

$$
-R^{a b c d} R_{a}^{e f g}{ }_{; e} R_{b c f}{ }_{; h}^{h}{ }_{h d} \equiv-I_{\{0,1,3\}, 2595} .
$$

This process is very fast: see Figure 1 for an histogram of timings of a case with 28 indices. In this way, we enormously reduce the number of invariants we need to control. There are $16 ! \sim 2 \cdot 10^{13}$ invariants for case $\{0,1,3\}$. As shown in Table 1 , there are 3237 different canonical forms (column 'Canon'), and if we remove products of invariants of lower degree, this number is further reduced to 3099 (column 'Invars'), which are indexed from $I_{\{0,1,3\}, 1}$ to $I_{\{0,1,3\}, 3099}$. The whole column 'Invars' has been constructed by canonicalization of a supercomplete set of more than 20 million permutations in 15 hours, giving 640119 different nondual canonical forms and 7698 canonical duals. Those canonical forms were then sorted within each case according to the following priorities: i) invariants with more (differentiated) Ricci scalars are sorted first; ii) invariants with more Ricci tensors are sorted first; iii) more Laplacians first; iv) ordering based on indices. The indexing of algebraic invariants in the first version of Invar has been preserved for backwards compatibility.

We do not consider at this step symmetries coming from permutation of covariant derivatives acting on scalar expressions. They will be treated in step 4.

(2) Cyclic symmetry. The Riemann tensor obeys the multiterm symmetry $R_{a[b c d]}=0$. For each canonical invariant after step 1 we generate several cyclic relations by replacing $R_{a b c d}$ by its 3 -index antisymmetrized part in all possible inequivalent ways. For example, from the canonical form (3) we get three cyclic relations (already canonicalized):

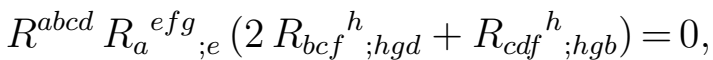

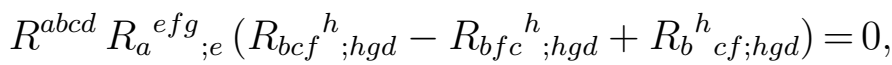

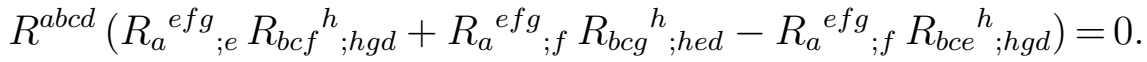




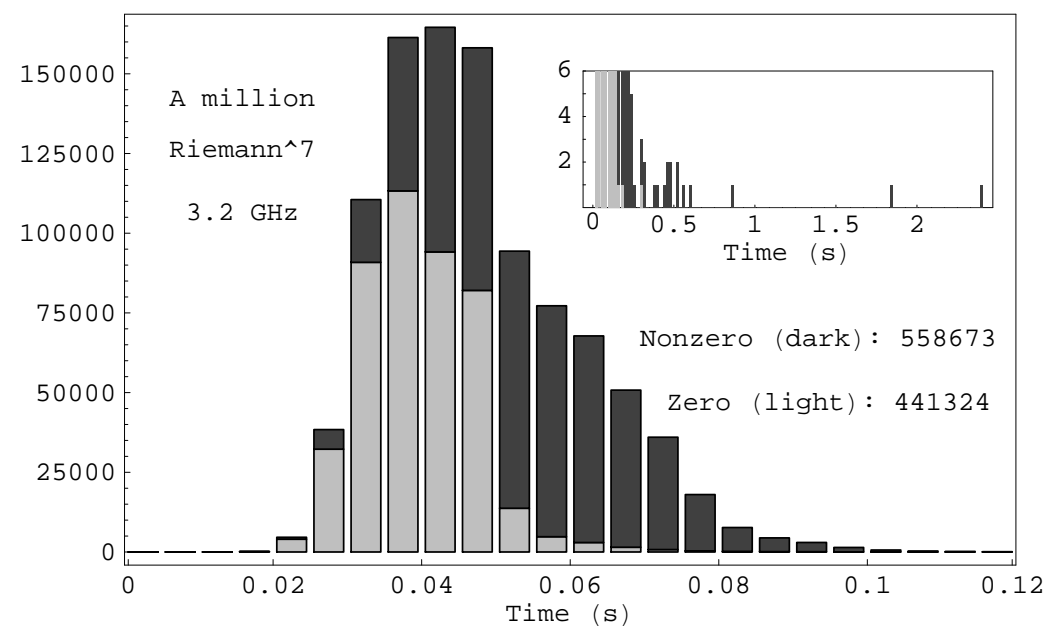

Fig. 1. Histogram of timings of canonicalization with xTensor [11] of a million algebraic invariants of degree 7 . The average is below $0.05 \mathrm{~s}$ and only two cases (with exceptionally high symmetry groups involved) take more than a second.

In terms of indexed invariants those are respectively

$$
\begin{array}{r}
2 I_{\{0,1,3\}, 2595}+I_{\{0,1,3\}, 2610}=0, \\
I_{\{0,1,3\}, 2595}-I_{\{0,1,3\}, 2601}+I_{\{0,1,3\}, 2757}=0, \\
I_{\{0,1,3\}, 2595}-I_{\{0,1,3\}, 2634}+I_{\{0,1,3\}, 2640}=0,
\end{array}
$$

allowing to express $I_{\{0,1,3\}, 2610}, I_{\{0,1,3\}, 2757}$ and $I_{\{0,1,3\}, 2640}$ in terms of lowerindex invariants.

(3) Bianchi identity. The Riemann tensor obeys the multiterm symmetry $R_{a b[c d ; e]}=0$. (Note that this, together with the previous permutation and cyclic symmetries, make the tensor $R_{a b c d ; e}$ vanish under antisymmetrization of any set of 3, 4 or 5 indices.) Again, from every one of the independent invariants after step 1, two equations are constructed by replacing $\nabla_{a} R_{b c d e}$ by $\nabla_{[a} R_{b c] d e}$ and $\nabla_{[a} R_{d e] b c}$. The results are then simplified using the information of steps 1 and 2 . For example from the canonical invariant (3) we get a zero equation and

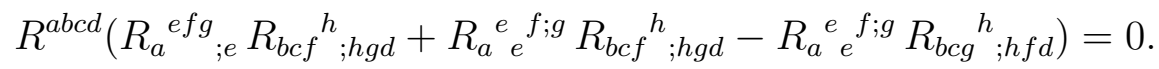

In terms of indexed invariants and using step 2 this is

$$
I_{\{0,1,3\}, 1445}-I_{\{0,1,3\}, 1451}+I_{\{0,1,3\}, 2595}=0,
$$

so that $I_{\{0,1,3\}, 2595}$ is no longer an independent invariant.

(4) Commutation of derivatives. Given any tensor $T^{a_{1} \ldots a_{n}} b_{1} \ldots b_{m}$ we have

$$
\begin{aligned}
& \nabla_{d} \nabla_{c} T_{b_{1} \ldots b_{m}}^{a_{1} \ldots a_{n}}-\nabla_{c} \nabla_{d} T_{b_{1} \ldots b_{m}}^{a_{1} \ldots a_{n}}= \\
& =\sum_{k=1}^{n} R_{c d e}{ }^{a_{k}} T_{b_{1} \ldots b_{m}}^{a_{1} \ldots e \ldots a_{n}}-\sum_{k=1}^{m} R_{c d b_{k}}{ }^{e} T_{b_{1} \ldots e \ldots b_{m}}^{a_{1} \ldots a_{n}}
\end{aligned}
$$


We generate new equations by exchanging the order of all consecutive covariant derivatives in the canonical invariants after step 1. As we have already mentioned, in this step different cases are mixed because second covariant derivatives are converted into Riemann tensors, and so we find equations involving cases with the same order $\Lambda$ but larger degree, that is $\{0,1,3\}$ and $\{0,0,1,1\}$ in our example (3): under commutation of $\nabla_{g}$ and $\nabla_{d}$ and after the use of steps 1,2 and 3 we get

$$
\begin{gathered}
-I_{\{0,1,3\}, 535}+I_{\{0,1,3\}, 536}+I_{\{0,1,3\}, 538}-I_{\{0,1,3\}, 539} \\
+I_{\{0,1,3\}, 541}-I_{\{0,1,3\}, 542}-I_{\{0,1,3\}, 547}+I_{\{0,1,3\}, 548} \\
+I_{\{0,0,1,1\}, 385}-I_{\{0,0,1,1\}, 386}-I_{\{0,0,1,1\}, 394}+I_{\{0,0,1,1\}, 397}=0 .
\end{gathered}
$$

The equations at this step, once expanded in terms of independent invariants, become very long, many of them having several thousand terms for $\Lambda=12$. This is because in step 4 we still have 6368 independent invariants, without even counting the 1639 objects from the algebraic degree- 7 case. The hardest case to build is $\{10\}$, taking nearly 300 hours of CPU time and containing equations with up to 5617 terms (independent invariants).

Our results in columns 'Commute' of tables 1 and 2 perfectly coincide with those of column 'Total' of appendix A of Fulling et al. [9] after dealing with the fact that they count product invariants. Note that they do not study dual invariants and so everything in table 3 is new.

(5) Dimensionally dependent identities (also called Lovelock type identities): antisymmetrization in $d+1$ indices in dimension $d$ gives zero [13]. We generate a large number of those equations for dimension 4 by antisymmetrization of random groups of 5 indices in each independent invariant after step 1.

Again, our results in column '4D' of tables 1 and 2 agree with those of Fulling et al. [9]. They give no results for dual invariants.

(6) Duals. A product of two $\epsilon$ tensors can be given as a linear combination of products of $\delta$ 's and hence a product of two dual invariants can be expressed as a linear combination of nondual invariants. We have combined the dual invariants in pairs in all possible ways, obtaining 51 relations which allow decomposing 51 independent invariants after step 5 into products of dual invariants.

We have chosen this order of use of symmetries because only steps 1-5 are signature independent, only steps 1-4 are dimension independent, steps 1-3 do not mix cases, steps 1-2 do not involve derivatives, and finally step 1 employs only permutation symmetries, which must be used first. Note the importance of not decomposing Riemann in its Weyl and Ricci parts, which would make the process dimension-dependent right from the outset. 


\begin{tabular}{|l|ccccccc|}
\hline Case & Canon & Invars & Cyclic & Bianchi & Commute & 4 D & Duals \\
\hline$\{0\}$ & 1 & 1 & 1 & 1 & 1 & 1 & 1 \\
\hline$\{0,0\}$ & 4 & 3 & 2 & 2 & 2 & 2 & 2 \\
$\{2\}$ & 2 & 2 & 2 & 1 & 1 & 1 & 1 \\
\hline$\{0,0,0\}$ & 13 & 9 & 5 & 5 & 5 & 3 & 3 \\
$\{0,2\}$ & 14 & 12 & 9 & 5 & 3 & 3 & 3 \\
$\{1,1\}$ & 12 & 12 & 9 & 4 & 4 & 4 & 4 \\
$\{4\}$ & 12 & 12 & 11 & 6 & 1 & 1 & 1 \\
\hline$\{0,0,0,0\}$ & 57 & 38 & 15 & 15 & 15 & 4 & 3 \\
$\{0,0,2\}$ & 119 & 99 & 48 & 27 & 15 & 10 & 10 \\
$\{0,1,1\}$ & 137 & 125 & 63 & 23 & 23 & 17 & 17 \\
$\{0,4\}$ & 138 & 126 & 84 & 47 & 3 & 3 & 3 \\
$\{1,3\}$ & 138 & 138 & 95 & 32 & 5 & 5 & 5 \\
$\{2,2\}$ & 89 & 86 & 59 & 23 & 7 & 7 & 7 \\
$\{6\}$ & 105 & 105 & 90 & 50 & 1 & 1 & 1 \\
\hline$\{0,0,0,0,0\}$ & 288 & 204 & 54 & 54 & 54 & 5 & 3 \\
$\{0,0,0,2\}$ & 1193 & 1020 & 313 & 175 & 79 & 26 & 25 \\
$\{0,0,1,1\}$ & 1922 & 1749 & 564 & 194 & 194 & 76 & 74 \\
$\{0,0,4\}$ & 1647 & 1473 & 648 & 361 & 17 & 12 & 12 \\
$\{0,1,3\}$ & 3237 & 3099 & 1387 & 442 & 53 & 42 & 42 \\
$\{0,2,2\}$ & 1735 & 1622 & 727 & 244 & 46 & 34 & 34 \\
$\{1,1,2\}$ & 1641 & 1617 & 741 & 143 & 67 & 52 & 52 \\
$\{0,6\}$ & 1770 & 1665 & 1025 & 570 & 3 & 3 & 3 \\
$\{1,5\}$ & 1770 & 1770 & 1115 & 362 & 5 & 5 & 5 \\
$\{2,4\}$ & 1770 & 1746 & 1093 & 356 & 9 & 9 & 9 \\
$\{3,3\}$ & 962 & 962 & 612 & 211 & 9 & 9 & 9 \\
$\{8\}$ & 1155 & 1155 & 945 & 525 & 1 & 1 & 1 \\
\hline
\end{tabular}

Table 1

Number of independent non-dual invariants after the different steps of simplification: 0) 'Canon': canonical invariants including products of lower degree; 1) 'Invars': canonical invariants without products; 2) 'Cyclic': invariants after imposing the Cyclic symmetry; 3) 'Bianchi': invariants after imposing the Bianchi identity; 4) 'Commute': invariants after commuting covariant derivatives; 5) '4D': invariants after imposing all possible dimensionally dependent identities for dimension 4; and 6) 'Duals': independent invariants after decomposition in products of duals.

\section{Implementation}

The implementation of the new database of relations among the differential invariants closely follows that of our previous version of Invar [1]. In particular the commands given in appendices $\mathrm{C}$ and $\mathrm{D}$ of [1] are still valid, with minimal changes, the most important of them being the fact that now the different cases are identified by the case list of orders $\{0,1,3\}$ in the Mathematica version and $[0,1,3]$ in the Maple version rather than an integer degree. Therefore an invariant is now denoted as $\operatorname{RInv}[\{0,1,3\}, 2595]$ or DualRInv $[\{1,3\}$, 920] in Mathematica and RInv [ [0,1,3], 2595] or DualRInv [ [1,3], 920] 


\begin{tabular}{|l|ccccccc|}
\hline Case & Canon & Invars & Cyclic & Bianchi & Commute & 4 D & Duals \\
\hline$\{0,0,0,0,0,0\}$ & 2070 & 1613 & 270 & 270 & 270 & 8 & 4 \\
$\{0,0,0,0,2\}$ & 14408 & 12722 & 2495 & 1371 & 549 & 66 & 58 \\
$\{0,0,0,1,1\}$ & 29427 & 27022 & 5439 & 1725 & 1725 & 245 & 228 \\
$\{0,0,0,4\}$ & 21750 & 19617 & 5622 & 3094 & 99 & 37 & 36 \\
$\{0,0,1,3\}$ & 64635 & 60984 & 17662 & 5440 & 577 & 242 & 240 \\
$\{0,0,2,2\}$ & 33252 & 30974 & 9030 & 2861 & 445 & 169 & 165 \\
$\{0,1,1,2\}$ & 64500 & 62465 & 18272 & 3226 & 1235 & 505 & 503 \\
$\{1,1,1,1\}$ & 5684 & 5606 & 1733 & 210 & 210 & 86 & 83 \\
$\{0,0,6\}$ & 27675 & 25590 & 10600 & 5840 & 17 & 12 & 12 \\
$\{0,1,5\}$ & 54930 & 53160 & 22330 & 6938 & 55 & 44 & 44 \\
$\{0,2,4\}$ & 54930 & 52764 & 22063 & 6861 & 93 & 72 & 72 \\
$\{1,1,4\}$ & 27540 & 27396 & 11695 & 2121 & 83 & 66 & 66 \\
$\{0,3,3\}$ & 27986 & 27024 & 11402 & 3597 & 68 & 53 & 53 \\
$\{1,2,3\}$ & 54930 & 54654 & 23255 & 4204 & 212 & 171 & 171 \\
$\{2,2,2\}$ & 9280 & 9104 & 3879 & 715 & 66 & 49 & 49 \\
$\{0,8\}$ & 26670 & 25515 & 14910 & 8225 & 3 & 3 & 3 \\
$\{1,7\}$ & 26670 & 26670 & 15855 & 5000 & 5 & 5 & 5 \\
$\{2,6\}$ & 26670 & 26460 & 15675 & 4950 & 9 & 9 & 9 \\
$\{3,5\}$ & 26670 & 26670 & 15855 & 5000 & 11 & 11 & 11 \\
$\{4,4\}$ & 13685 & 13607 & 8111 & 2615 & 12 & 12 & 12 \\
$\{10\}$ & 15120 & 15120 & 11970 & 6615 & 1 & 1 & 1 \\
\hline$\{0,0,0,0,0,0,0\}$ & 19610 & 16532 & 1639 & 1639 & 1639 & 7 & 3 \\
\hline
\end{tabular}

Table 2

Number of independent invariants for the cases with order $\Lambda=12$, plus the algebraic case of degree 7 . See caption of table 1 for the meaning of the column headers.

\begin{tabular}{|l|cccccc|}
\hline Dual case & Canon & Invars & Cyclic & Bianchi & Commute & 4 D \\
\hline$\{0\}^{*}$ & 1 & 1 & 0 & 0 & 0 & 0 \\
\hline$\{0,0\}^{*}$ & 5 & 4 & 1 & 1 & 1 & 1 \\
$\{2\}^{*}$ & 3 & 3 & 0 & 0 & 0 & 0 \\
\hline$\{0,0,0\}^{*}$ & 35 & 27 & 6 & 6 & 6 & 2 \\
$\{0,2\}^{*}$ & 63 & 58 & 13 & 5 & 1 & 1 \\
$\{1,1\}^{*}$ & 36 & 36 & 9 & 2 & 2 & 2 \\
$\{4\}^{*}$ & 32 & 32 & 11 & 4 & 0 & 0 \\
\hline$\{0,0,0,0\}^{*}$ & 288 & 232 & 40 & 40 & 40 & 1 \\
$\{0,0,2\}^{*}$ & 1059 & 967 & 212 & 98 & 29 & 6 \\
$\{0,1,1\}^{*}$ & 1095 & 1047 & 236 & 54 & 54 & 13 \\
$\{0,4\}^{*}$ & 920 & 876 & 285 & 128 & 1 & 1 \\
$\{1,3\}^{*}$ & 920 & 920 & 296 & 60 & 2 & 2 \\
$\{2,2\}^{*}$ & 484 & 478 & 163 & 37 & 3 & 3 \\
$\{6\}^{*}$ & 435 & 435 & 220 & 95 & 0 & 0 \\
\hline$\{0,0,0,0,0\}^{*}$ & 3031 & 2582 & 330 & 330 & 330 & 2 \\
\hline
\end{tabular}

Table 3

Number of independent dual invariants after the different steps of simplification. See the caption of table 1 for the meaning of the column headers. Note that there is no final column 'Duals' in this table. 
in Maple. The old notation RInv [3, 9] for an algebraic invariant is automatically translated to its new notation $\operatorname{RInv}[\{0,0,0\}$, 9] in Mathematica and RInv $[[0,0,0], 9]$ in Maple.

The system now handles derivatives of tensorial expressions. The covariant derivative $\nabla_{a}$ is treated as an operator and denoted $\mathrm{CD}[-\mathrm{a}]$ in our tensor computer algebra systems. Hence, we can represent the invariant

$$
I_{\{1,1\}, 2} \equiv R_{a b}^{a b}{ }^{; c} R_{c}^{d}{ }_{d ; e}^{e}=-R_{; a}^{a b} R_{; b}
$$

as $\mathrm{CD}[\mathrm{c}][\mathrm{R}[\mathrm{a}, \mathrm{b},-\mathrm{a},-\mathrm{b}]] * \mathrm{CD}[-\mathrm{e}][\mathrm{R}[-\mathrm{c}, \mathrm{d},-\mathrm{c}, \mathrm{e}]]$ in Mathematica, and as $\mathrm{CD}[\mathrm{c}](\mathrm{R}[\mathrm{a}, \mathrm{b},-\mathrm{a},-\mathrm{b}]) * \mathrm{CD}[-\mathrm{e}](\mathrm{R}[-\mathrm{c}, \mathrm{d},-\mathrm{c}, \mathrm{e}])$ in Maple.

Covariant derivatives in $x$ Tensor and Canon are linear operators and automatically obey the standard rules [14], in particular the Leibnitz rule for products. The canonicalization algorithms consistently manipulate derivatives and only one comment is required, concerning how the different Riemann tensors are sorted in a given invariant: we follow the convention of having tensors with more covariant derivatives on the right to help the canonicalizer, which starts placing indices on the left. That is why we chose the convention $\lambda_{i} \leq \lambda_{i+1}$ for the cases $\left\{\lambda_{1}, \ldots, \lambda_{n}\right\}$, opposite to the choice by Fulling et al. [9].

As a simple example of use of the main command RiemannSimplify we can check the interesting relation

$$
R_{a}^{a b c d ; e} R_{b e}{ }^{f g ; h i} R_{c f g i ; h}=\frac{1}{8} R_{e}^{a b c d ; e} R_{a b}{ }^{f g ; h i} R_{c d f g ; i h},
$$

in which the rearrangement of the lower indices produces a factor $1 / 8$. This is rule number 2868 in the file of Bianchi relations among the invariants of case $\{2,2,2\}$. We can check it in Mathematica using the sentence

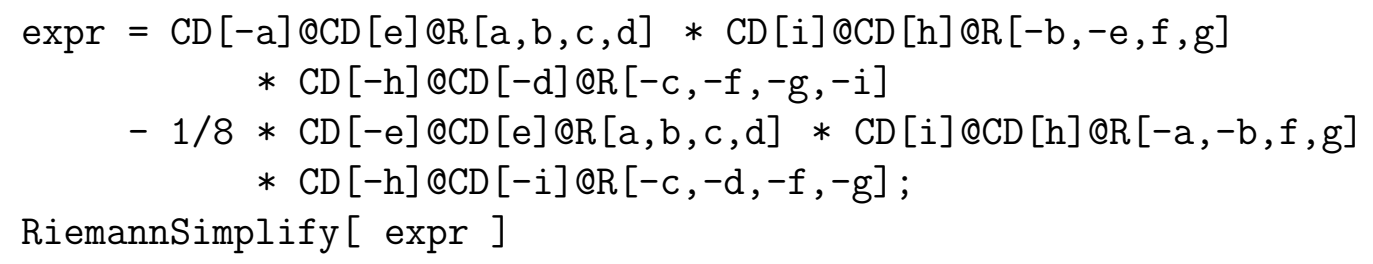

where $\mathrm{f} @ \mathrm{x}$ is a Mathematica shorthand for $\mathrm{f}[\mathrm{x}]$. The corresponding Maple expression can be obtained replacing each $f @ x$ by $f(x)$. The final call would be

RiemannSimplify ( expr) 
The database of relations is arranged as follows: there is a file for each step and case (so $6 \times 48+5 \times 15=363$ files in total) containing the relations at that step among the invariants of that case and all previous cases. The full database takes more than 1.5 Gbytes of memory and even more once it is read by the computer algebra systems. Most of it comes from the 13 last cases of order 12 at the commutation step (step 4 ), and so we provide alternative smaller files in which the rules have not been fully expanded in terms of independent invariants of previous cases. The user can configure the system to read either the fully expanded files or the non-expanded files in those cases. If the latter are chosen then repeated (up to four times) use of the simplification functions might be needed to fully expand an invariant, resulting in a slower process. With non-expanded files we have 365Mbytes of data.

\section{Conclusions}

We have successfully extended our tensor computer algebra package Invar to handle differential invariants of the Riemann tensor up to 12 derivatives of the metric. Unlike the problem of algebraic invariants, to which a great deal of effort has been dedicated in the last decades, the problem of differential invariants remained almost unexplored due to its much more difficult character: two new sources of equations come into play, namely the Bianchi identity and the non-commutation of covariant derivatives. This article has completely solved the problem for those cases most likely to be needed in current computations. The problem has been solved in the most useful way: by providing a database with all equations coming from multiterm symmetries, such that any invariant can be immediately looked up without the need of inefficient intermediate computations.

The new database is much larger than its previous version, now containing 645625 relations for 647817 canonical invariants (counting both dual and nondual invariants). This represents an increase by a factor larger than 30 with respect to the 21221 relations for 21246 invariants in the old database. But this investigation is not only a quantitative extension of [1]. We have also extended our algorithms to handle derivatives and their associated symmetries: Bianchi and commutation. The latter has been especially hard, producing equations with thousands of terms, such that the database has increased in size by a factor larger than 250 with respect to the previous version.

Invar runs on top of the multipurpose tensor computer algebra systems $x$ Tensor [11] for Mathematica and Canon [12] for Maple.

A final step is required to construct a computer algebra system for generic treatment of the Riemann tensor: manipulation of expression with free indices. We are currently analyzing this extension. 


\section{Acknowledgements}

JMM thanks Brian Edgar for helpful discussions and the University of Linköping for hospitality. JMM was supported by the Spanish MEC under the research project FIS2005-05736-C03-02. DY acknowledges the grant "Beca de introducción a la investigación" from CSIC. RP acknowledges grant no. 2898-07-1 from CAPES. Part of the computations were performed at the Centro de SuperComputación de Galicia (CESGA).

\section{References}

[1] J.M. Martín-García, R. Portugal and L.R.U. Manssur, Comp. Phys. Commun. 177 (2007) 640-648.

[2] G.E. Sneddon, J. Math. Phys. 40 (1999) 5905-5920.

[3] A.E.K. Lim and J. Carminati, J. Math. Phys. 48 (2007) 082503.

[4] W.B. Bonnor, Class. Quant. Grav. 11 (1994) 2007-2012.

[5] P. Musgrave and K. Lake, Class. Quant. Grav. 12 (1995) L39-L41.

[6] M.H. Goroff and A. Sagnotti, Nucl. Phys. B 266 (1986) 709-736.

[7] D. Wands, Class. Quant. Grav. 11 (1994) 269-280.

[8] V. Iyer and R.M. Wald, Phys. Rev. D 50 (1994) 846-864; 52 (1995) 4430-4439.

[9] S.A. Fulling et al., Class. Quant. Grav. 9 (1992) 1151-1197.

[10] L.R.U. Manssur, R. Portugal and B.F. Svaiter, Int. J. Modern Phys. C 13 (2002) 859-879.

[11] xTensor, A fast manipulator of tensor expressions, J. M. Martín-García 20022008, (http://metric.iem.csic.es/Martin-Garcia/xAct/)

[12] L.R.U. Manssur and R. Portugal, Comp. Phys. Commun. 157 (2004) 173-180. (http://www. lncc.br/ portugal/Canon.html)

[13] S.B. Edgar and A. Hoglund, J. Math. Phys. 43, (2002) 659-677.

[14] R.M. Wald, General Relativity, The University of Chicago Press, Chicago 1984. See page 31. 